\title{
Suplementos utilizados como hidratantes nas fases pré-alojamento e pós-alojamento para pintos recém eclodidos
}

\author{
Adriana Ayres Pedroso(1), José Henrique Stringhini(1), Nadja Susana Mogyca Leandro(1), Marcos Barcellos Café(1), \\ Carlos Eduardo Barbosa ${ }^{(1)}$ e Flávia Gontijo de Lima ${ }^{(1)}$
}

\begin{abstract}
(1)Universidade Federal de Goiás, Dep. de Produção Animal, Campus Samambaia, Rodovia Nova Veneza-Goiânia, Caixa Postal 131, CEP 74001-970 Goiânia, GO. E-mail: adrianaped@yahoo.com.br, jhstring@uol.com.br, mogyca@vet.ufg.br, mcafe@vet.ufg.br, flaviamedvet@yahoo.com.br, cadu@vet.gard.ufg.br
\end{abstract}

\begin{abstract}
Resumo - Dois experimentos foram conduzidos, para avaliar hidratantes em pintos oriundos de matrizes, em início de vida produtiva. O primeiro experimento estudou o efeito de hidratante comercial, na fase pré-alojamento, em pintos oriundos de matrizes com 25 semanas de idade, submetidos a dois intervalos entre nascimento e alojamento ( 0 e 48 horas); o agente não apresentou qualquer efeito favorável sobre a desidratação, a mortalidade e o desempenho até 21 dias. No segundo experimento, foi utilizado o bicarbonato de sódio como hidratante. A solução foi fornecida, durante 24 horas, a pintos oriundos de matrizes com 27 semanas de idade, após 0,24 e 48 horas de intervalo entre nascimento e alojamento. Foi observada melhora na integridade do intestino, representada pelo aumento na densidade intestinal e peso do órgão com a suplementação eletrolítica. O aumento no intervalo entre nascimento e alojamento diminuiu o saco vitelino. Em ambos os experimentos, o aumento no intervalo entre o nascimento e o alojamento aumentou a desidratação e a mortalidade das aves, mas propiciou melhora no desempenho aos 21 dias, não tendo sido observados efeitos positivos dos hidratantes testados.
\end{abstract}

Termos para indexação: alojamento, desidratação, eletrólito, qualidade de pinto.

\section{Supplements used as hydrating agents in pre- and post-lodging phases to newly-hatched chicks}

\begin{abstract}
Two experiments were carried out to evaluate hydrating agents to newly hatched chicks from breeders in early production life. The first experiment studied the effect of commercial hydrating agents in pre-lodging phase in chicks from breeders with 25 weeks of age and submitted to two intervals between hatching and lodging ( 0 and 48 hours). Agent did not show any favorable effects on dehydration, mortality and performance until 21 days of age. In he second experiment, sodium bicarbonate was used as hydrating agent. The solution was supplied for 24 hours, after 0,24 and 48 hours intervals between hatching and lodging, to chicks from breeder of 27 weeks of age. The intestinal integrity increased, based on intestinal density and organ weight increment obtained with electrolytes supplementation. As the interval between hatching and lodging increased, the yolk sac decreased. In both experiments, the interval increasing between hatching and lodging promoted higher dehydration and mortality incidence, but performance was better at 21 days of age.
\end{abstract}

Index terms: lodging, dehydration, electrolyte, chick quality.

\section{Introdução}

A importância da qualidade dos pintos de corte é freqüentemente subestimada, em relação à manifestação do potencial genético da ave (Salazar, 2003); calcula-se que pintos de má qualidade podem chegar a custar 200 milhões de dólares à indústria avícola (Uni, 2003). A boa qualidade dos pintos que chegam à granja comercial é fundamental, para que seja alcançado um ótimo desempenho ao final da criação. Peso adequado, acima de 40 g, e animais hidratados podem ser interpretados como característicos de aves de qualidade (Cunha, 2003).

A chegada de pintos de peso e qualidade reduzida em granjas é característica de épocas de crise no setor avícola. A alta demanda força empresas a incubar ovos de tamanho reduzido, oriundos de matrizes em início de postura, que podem originar animais pequenos. Pode ocorrer alta mortalidade por desidratação, principalmente se os pintos forem transportados por longas distâncias, 
em regiões quentes, o que compromete o trato digestivo e conseqüentemente o desempenho (Daghir \& Jones, 1997; Baião \& Aguilar, 2001).

Uma estratégia adotada para minimizar perdas na qualidade de pintos é o fornecimento de hidratantes, antes ou após o alojamento. Em relação ao desempenho e mortalidade, respostas positivas ao uso de soluções à base de eletrólitos essenciais, facilmente manipuladas, podem ser conseqüência da homeostase da ave (Laurentiz et al., 2000). Empresas fornecem, comercialmente, hidratantes a serem oferecidos nas embalagens de transporte, que aliviam os efeitos da perda de água, no percurso entre o incubatório e a granja de criação. Porém, essa terapia de reidratação carece de informações baseadas em literatura científica (Baião \& Borges, 1995).

Este trabalho foi desenvolvido para avaliar o efeito de agentes hidratantes sobre a perda de peso, a mortalidade, o desempenho e a biometria de órgãos relacionados ao sistema digestório de pintos, de tamanho reduzido, submetidos a diferentes intervalos entre o nascimento e o alojamento.

\section{Material e Métodos}

Os experimentos foram realizados no Aviário Experimental, do Departamento de Produção Animal, da Escola de Veterinária, da Universidade Federal de Goiás, no período de março a maio de 2004.

O experimento 1 foi desenvolvido em delineamento de blocos ao acaso, em esquema fatorial $2 \times 2$ (intervalos de alojamento de 0 e 48 horas x oferecimento ou não de hidratante comercial pré-alojamento), com quatro repetições de oito aves por tratamento. $\mathrm{O}$ fator sexo foi utilizado para a determinação dos blocos. Foi utilizado o hidratante Oasis ${ }^{\circledR}$, comercializado pela Novus Internacional, tendo sido fornecido $2 \mathrm{~g}$ de produto por ave, para cada período de 24 horas de embalagem em caixa de papelão. Conforme informação dos fabricantes, o produto contém $75 \%$ de umidade, $20 \%$ de proteína bruta, $0,5 \%$ de extrato etéreo e $3 \%$ de fibra bruta. As aves foram mantidas em conforto térmico nas dependências do incubatório. Foram utilizados pintos de corte, da linhagem Cobb, oriundos de matrizes com 25 semanas de idade, com peso médio de 32,9 g.

No experimento 2, o delineamento adotado foi o de blocos ao acaso, em esquema fatorial 3x2 (intervalo de alojamento de 0 , 24 e 48 horas x oferecimento ou não de solução eletrolítica pós-alojamento), com quatro re- petições de oito aves por tratamento. Foram constituídos dois blocos de fêmeas e dois de machos. Foram utilizados pintos oriundos de matrizes da linhagem Cobb, com 27 semanas de idade, com peso médio de 38,9 g ao nascimento. As aves foram embaladas em caixas de papelão pelo período determinado, mantidas em conforto térmico nas dependências do incubatório, e após o alojamento foi fornecida, durante 24 horas, solução eletrolítica à base de bicarbonato de sódio, de modo que o Na, Cl e K da dieta e da solução perfizessem 250 mEq (Mongin, 1981).

Em ambos os experimentos, foram avaliados: o peso das aves no momento da embalagem e do alojamento, a porcentagem de perda de peso em função do peso inicial, a mortalidade nas primeiras 48 horas e o desempenho zootécnico, semanalmente, até 21 dias de idade. No segundo experimento, 24 horas após o alojamento, uma ave por parcela era sacrificada e era avaliado o peso do esôfago + papo, proventículo + moela, intestino delgado, intestino grosso, pâncreas, fígado e saco vitelino. O comprimento do intestino delgado foi tomado para a avaliação da densidade do órgão, expressa em gramas por centímetro. A mortalidade foi transformada em arc sen, para análise estatística. Os dados foram analisados pelo PROC GLM do SAS (1998) e comparados pelo teste de Tukey.

\section{Resultados e Discussão}

No experimento 1, o peso dos animais no momento do alojamento diminuiu à medida que aumentou o tempo que as aves permaneceram na caixa de papelão; o mesmo ocorreu com a porcentagem de perda de peso, que apresentou efeito significativo (Tabela 1). O suplemento comercial não foi eficiente para prevenir a desidratação das aves, traduzida como a perda de peso sofrida. Resultados semelhantes foram obtidos no experimento 2 , para o peso no momento do alojamento e porcentagem de perda de peso (Tabela 2). A diminuição no peso está relacionada com queda na qualidade do pinto (Moran Junior \& Reinhart, 1980). Em pesquisas que avaliaram a porcentagem de perda de peso da ave, foi verificado que as aves alojadas por 48 horas podem perder de $10 \%$ (Pinchasov \& Moy, 1993) a 21\% (Baião \& Cançado, 1998) do peso corpóreo, após um intervalo entre o nascimento e o alojamento de 48 horas. Nesse experimento foram obtidos valores de perda de peso intermediários, de aproximadamente $12 \%$, quando os pintos permaneceram 48 horas embalados. A perda no peso das aves 
pode ocorrer em função da perda de água, da absorção do saco vitelino e da perda da proteína muscular, que pode ser utilizada para neoglicogênese. Não houve influência do sexo da ave sobre a perda de peso, em ambos os experimentos.

A mortalidade observada até 48 horas após o alojamento foi grande, no experimento desenvolvido com pintos originados de matrizes com 25 semanas de idade (Tabela 3). Os animais ficaram debilitados e com dificuldade de ingerir água e ração, o que pode explicar a mortalidade observada no tempo zero, 48 horas após o alojamento, e no período total. O hidratante comercial fornecido na embalagem das aves não foi eficiente para reduzir a mortalidade, como preconizado pelos fabricantes do agente. No experimento 2, quando a mortalidade foi estudada em aves oriundas de matrizes apenas duas semanas mais velhas, os índices observados foram muitos baixos (Tabela 4). Não houve mortalidade alguma no momento de alojamento das aves nos boxes experi-

Tabela 1. Peso no momento da embalagem (PE) e do alojamento (PA), e perda de peso (PP), em pintos oriundos de matrizes com 25 semanas de idade, suplementados com hidratante na fase pré-alojamento ${ }^{(1)}$.

\begin{tabular}{lccc}
\hline Variáveis & PE $(\mathrm{g})$ & PA $(\mathrm{g})$ & PP $(\%)$ \\
\hline Intervalo de alojamento (horas) & & & \\
$\quad 0$ & 30,30 & $30,30 \mathrm{a}$ & 0,00 \\
$\quad 28$ & 29,96 & $25,78 \mathrm{~b}$ & 12,01 \\
\hline Hidratante pré-alojamento & & & \\
$\quad$ Ausência & 30,10 & 25,82 & 14,03 \\
$\quad$ Presença & 30,02 & 28,02 & 6,95 \\
\hline Sexo & & & \\
$\quad$ Macho & 29,50 & 26,68 & 9,56 \\
$\quad$ Fêmea & 30,65 & 27,89 & 9,06 \\
\hline CV (\%) & 5,01 & 5,54 & 3,66 \\
\hline
\end{tabular}

(1)Médias seguidas de letras iguais não diferem, estatisticamente, a $1 \%$ de probabilidade, pelo teste de Tukey.

Tabela 2. Peso no momento da embalagem (PE) e do alojamento (PA), e perda de peso (PP), em pintos oriundos de matrizes com 27 semanas de idade, suplementados com hidratante na fase pós-alojamento ${ }^{(1)}$.

\begin{tabular}{lrrr}
\hline Variáveis & PE $(\mathrm{g})$ & PA $(\mathrm{g})$ & PP $(\%)$ \\
\hline Intervalo de alojamento (horas) & & & \\
0 & 30,30 & $30,30 \mathrm{a}$ & 0,00 \\
24 & 29,96 & $27,24 \mathrm{~b}$ & 6,73 \\
48 & 29,20 & $25,78 \mathrm{~b}$ & 13,97 \\
\hline Sexo & & & \\
Macho & 29,51 & 27,01 & 8,47 \\
Fêmea & 29,94 & 27,53 & 8,09 \\
\hline CV $(\%)$ & 4,80 & 4,53 & 5,03 \\
\hline
\end{tabular}

(1)Médias seguidas de letras iguais não diferem, estatisticamente, a $2 \%$ de probabilidade, pelo teste de Tukey. mentais, bem como após 24 horas. Possivelmente, a idade da ave levou a uma melhor condição corporal e resultou em menores baixas nesse lote experimental. A suplementação eletrolítica, realizada 0 e 24 horas após o alojamento, e o sexo da ave não tiveram influência sobre os resultados obtidos.

Para efeito de cálculo, os índices relacionados ao desempenho foram mencionados considerando-se o dia de alojamento da ave como o primeiro dia de idade, a exemplo do que ocorre no campo. Além disso, com a finalidade de comparar dados, o dia de nascimento da ave também foi considerado como o primeiro dia de idade. O desempenho de frangos após 21 dias de nascimento, ou de alojamento, não foi influenciado pela suplementação hidratante oferecida nas fases pré-alojamento ou pós-alojamento nos experimentos 1 e 2 (Tabelas 5 e 6). Resultados similares foram obtidos por Baião et al. (1998), que utilizaram solução eletrolítica, e por Tanaka \& Xin (1997), que pesquisaram o hidratante comercial. Embora alguns trabalhos demonstrem o efeito positivo de hidratantes sobre o desempenho (Xin \& Lee, 1996; Batal \& Parson, 2002), no presente experimento, tal efeito não foi observado.

Tabela 3. Mortalidade observada após 0, 24 e 48 horas de alojamento e no período total (0-48 horas), em pintos oriundos de matrizes com 25 semanas de idade, suplementados com hidratante na fase pré-alojamento ${ }^{(1)}$.

\begin{tabular}{|c|c|c|c|c|}
\hline Variáveis & $0(\%)$ & $24(\%)$ & $48(\%)$ & $0-48(\%)$ \\
\hline \multicolumn{5}{|c|}{ Intervalo de alojamento (horas) } \\
\hline 0 & $0,00 \mathrm{a}$ & $0,04 \mathrm{a}$ & 0,00 & $0,04 \mathrm{a}$ \\
\hline 48 & $0,08 \mathrm{~b}$ & $0,41 \mathrm{~b}$ & 0,17 & $0,73 b$ \\
\hline \multicolumn{5}{|c|}{ Hidratante pré-alojamento } \\
\hline Ausência & 0,03 & 0,29 & 0,13 & 0,52 \\
\hline Presença & 0,10 & 0,27 & 0,07 & 0,48 \\
\hline \multicolumn{5}{|l|}{ Sexo } \\
\hline Macho & 0,07 & 0,35 & 0,05 & 0,44 \\
\hline Fêmea & 0,04 & 0,23 & 0,17 & 0,55 \\
\hline $\mathrm{CV}(\%)$ & 155,78 & 91,61 & 158,09 & 49,87 \\
\hline
\end{tabular}

Tabela 4. Mortalidade observada após 48 horas de alojamento e no período total (0-48 horas), em pintos oriundos de matrizes com 27 semanas de idade, suplementados com hidratante na fase pós-alojamento.

\begin{tabular}{lrr}
\hline Variáveis & $48(\%)$ & $0-48(\%)$ \\
\hline Intervalo de alojamento (horas) & & \\
0 & 0,00 & 0,00 \\
24 & 0,00 & 0,00 \\
48 & 0,04 & 0,04 \\
\hline Sexo & & \\
Macho & 0,01 & 0,01 \\
Fêmea & 0,01 & 0,01 \\
\hline CV $(\%)$ & 346,41 & 346,41 \\
\hline
\end{tabular}


No experimento desenvolvido com pintos oriundos de matrizes com 25 semanas de idade, a conversão alimentar foi melhor, e o consumo de ração menor, após 48 ho-

Tabela 5. Peso médio (PM), consumo de ração (CR) e conversão alimentar (CA) após 21 dias de alojamento, ou de nascimento, de frangos oriundos de matrizes com 25 semanas de idade, suplementados com hidratante na fase pré-alojamento ${ }^{(1)}$.

\begin{tabular}{|c|c|c|c|c|c|}
\hline \multirow[t]{2}{*}{ Variáveis } & \multicolumn{3}{|c|}{ Após o alojamento } & \multicolumn{2}{|c|}{ Após o nascimento } \\
\hline & PM (g) & CR (g) & CA & PM (g) & CR (g) CA \\
\hline \multicolumn{6}{|c|}{ Intervalo de alojamento (horas) } \\
\hline 0 & 634 & $1.859 \mathrm{a}$ & $3,13 \mathrm{a}$ & 634 & $1.859 \mathrm{a} 3,13 \mathrm{a}$ \\
\hline 48 & 619 & $1.114 \mathrm{~b}$ & $2,02 \mathrm{~b}$ & 504 & $1.555 \mathrm{~b} 3,47 \mathrm{~b}$ \\
\hline \multicolumn{6}{|l|}{ Hidratante } \\
\hline Ausência & 638 & 1.720 & 2,88 & 597 & $1.781 \quad 3,08$ \\
\hline Presença & 605 & 1.101 & 2,05 & 495 & $1.576 \quad 3,61$ \\
\hline \multicolumn{6}{|l|}{ Sexo } \\
\hline Macho & 631 & 1.393 & 2,29 & 559 & $1.722 \quad 3,32$ \\
\hline Fêmea & 619 & 1.511 & 2,79 & 553 & $1.650 \quad 3,31$ \\
\hline $\mathrm{CV}(\%)$ & 19,36 & 7,17 & 17,02 & 19,84 & $9,08 \quad 22,95$ \\
\hline
\end{tabular}

(1)Médias seguidas de letras iguais não diferem, estatisticamente, a 5\% de probabilidade, pelo teste de Tukey.

Tabela 6. Peso médio (PM), consumo de ração (CR) e conversão alimentar (CA) após 21 dias de alojamento ou de nascimento, de frangos oriundos de matrizes com 27 semanas de idade, suplementados com hidratante após o alojamento ${ }^{(1)}$.

\begin{tabular}{|c|c|c|c|c|c|c|}
\hline \multirow[t]{2}{*}{ Variáveis } & \multicolumn{3}{|c|}{ Após o alojamento } & \multicolumn{3}{|c|}{ Após o nascimento } \\
\hline & PM (g) & CR (g) & $\mathrm{CA}$ & $\overline{\mathrm{PM}(\mathrm{g})}$ & CR $(\mathrm{g})$ & $\mathrm{CA}$ \\
\hline \multicolumn{7}{|c|}{ Intervalo de alojamento (horas) } \\
\hline 0 & 738 & $1.548 \mathrm{a}$ & $2,26 \mathrm{a}$ & $2,26 \mathrm{~b}$ & $1.548 \mathrm{~b}$ & 738 \\
\hline 24 & 745 & $1.272 \mathrm{c}$ & $1,80 \mathrm{~b}$ & $2,61 \mathrm{a}$ & $1.691 \mathrm{a}$ & 681 \\
\hline 48 & 729 & $1.339 \mathrm{~b}$ & $1,98 \mathrm{~b}$ & $2,85 \mathrm{a}$ & $1.616 \mathrm{ab}$ & 604 \\
\hline \multicolumn{7}{|l|}{ Eletrólitos } \\
\hline Ausência & 743 & 1.378 & 1,99 & 2,53 & 1.637 & 679 \\
\hline Presença & 731 & 1.396 & 2,03 & 2,62 & 1.600 & 669 \\
\hline \multicolumn{7}{|l|}{ Sexo } \\
\hline Macho & $779 \mathrm{a}$ & 1.408 & $1,92 \mathrm{a}$ & $2,47 \mathrm{a}$ & 1.639 & $709 \mathrm{a}$ \\
\hline Fêmea & $695 \mathrm{~b}$ & 1.365 & $2,10 \mathrm{~b}$ & $2,67 \mathrm{~b}$ & 1.598 & $640 \mathrm{~b}$ \\
\hline CV (\%) & 6,75 & 3,67 & 9,42 & 9,10 & 3,86 & 6,69 \\
\hline
\end{tabular}

(1)Médias seguidas de letras iguais não diferem, estatisticamente, a 5\% de probabilidade, pelo teste de Tukey. ras de alojamento (Tabela 5). Aves nascidas e imediatamente alojadas podem não apresentar boa conversão alimentar, pois têm uma fonte endógena de nutrição. $\mathrm{O}$ intervalo entre a embalagem e o alojamento pode ter propiciado melhores resultados, pois a ave já apresentava absorção parcial do saco vitelino, o que pode ter conduzido ao melhor aproveitamento da dieta ingerida. Analisando-se os dados com base no dia de nascimento da ave como o primeiro dia de idade, observa-se que a conversão alimentar foi melhor, e o consumo de ração menor, após 48 horas de alojamento. Não houve diferença no peso médio da ave. $\mathrm{O}$ tamanho extremamente reduzido da ave, aproximadamente $33 \mathrm{~g}$, talvez tenha impossibilitado que o efeito do sexo fosse manifestado sobre as características zootécnicas analisadas.

Em pesquisa desenvolvida por Cançado (1999), constatou-se que pintos de boa qualidade, alojados com $24 \mathrm{e}$ 48 horas após a eclosão, já no terceiro dia eram mais pesados que os animais alojados no dia da eclosão. De modo similar ao verificado no experimento 1 , a conversão alimentar, considerando-se o primeiro dia de idade da ave como o dia de alojamento, foi melhor após 24 ou 48 horas de embalagem no experimento 2, desenvolvido com aves oriundas de matrizes com 27 semanas de idade (Tabela 6). Novamente, esse fato pode estar relacionado com a absorção do saco vitelino, que apresentou uma diminuição linear após 48 horas de alojamento (Tabela 7).

Quando se considerou o dia de nascimento como o primeiro dia de vida da ave, observou-se pior conversão alimentar após 24 ou 48 horas de alojamento, resultado esperado, pois as aves foram privadas de dieta durante um e dois dias, respectivamente. Em ambas as situações, os machos apresentaram melhor conversão alimentar do que as fêmeas.

Tabela 7. Peso vivo (PV), peso absoluto do esôfago + papo (EP), proventrículo + moela (PM), intestino delgado (ID) e grosso (IG), pâncreas (PA), fígado (FG), saco vitelino (SV), comprimento (CP) e densidade do intestino delgado (DD), de pintos oriundos de matrizes com 27 semanas de idade, suplementados com hidratante na fase pós-alojamento e 24 horas após o

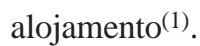

\begin{tabular}{|c|c|c|c|c|c|c|c|c|c|c|}
\hline Variáveis & $\mathrm{PV}(\mathrm{g})$ & $\mathrm{EP}(\mathrm{g})$ & PM (g) & ID $(\mathrm{g})$ & $\mathrm{IG}(\mathrm{g})$ & PA $(g)$ & $\mathrm{FG}(\mathrm{g})$ & SV $(g)$ & $\mathrm{CP}(\mathrm{cm})$ & $\mathrm{DD}\left(\mathrm{mg} \mathrm{cm}^{-1}\right)$ \\
\hline \multicolumn{11}{|c|}{ Intervalo de alojamento (horas) } \\
\hline 0 & $42,80 \mathrm{a}$ & 0,94 & 3,33 & 2,42 & 0,60 & 0,12 & 1,51 & 1,48 & $41,08 \mathrm{a}$ & 58,24 \\
\hline 24 & $39,20 \mathrm{a}$ & 1,24 & 2,88 & 2,23 & 0,65 & 0,14 & 1,43 & 0,65 & $42,50 \mathrm{a}$ & 52,20 \\
\hline 48 & $31,15 \mathrm{~b}$ & 0,62 & 2,44 & 1,65 & 0,63 & 0,10 & 1,09 & 0,42 & $35,20 \mathrm{~b}$ & 42,76 \\
\hline \multicolumn{11}{|l|}{ Eletrólitos } \\
\hline Ausência & 38,69 & 1,03 & 2,85 & 2,19 & 0,65 & 0,11 & 1,42 & 0,88 & 39,24 & 54,18 \\
\hline Presença & 36,74 & 0,84 & 2,92 & 2,00 & 0,61 & 0,13 & 1,26 & 0,94 & 40,28 & 48,91 \\
\hline \multicolumn{11}{|l|}{ Sexo } \\
\hline Macho & 39,14 & 1,02 & 2,74 & 2,20 & 0,64 & 0,11 & 1,34 & 0,89 & 40,15 & 53,45 \\
\hline Fêmea & 36,29 & 0,84 & 3,02 & 2,00 & 0,62 & 0,13 & 1,35 & 0,94 & 39,45 & 49,57 \\
\hline $\mathrm{CV}(\%)$ & 11,61 & 68,64 & 23,84 & 33,35 & 20,44 & 40,06 & 25,01 & 41,35 & 10,27 & 27,23 \\
\hline
\end{tabular}

${ }^{(1)}$ Médias seguidas de letras iguais não diferem, estatisticamente, a 5\% de probabilidade, pelo teste de Tukey. 
Considerando-se o dia de alojamento como o primeiro dia de idade da ave, o consumo de ração em aves alojadas imediatamente após o nascimento foi o maior observado. Aves alojadas após 48 horas de embalagem apresentaram maior consumo de ração do que aves alojadas após 24 horas de embalagem. Esse fato pode estar relacionado à voracidade da ave, em virtude da privação do alimento e da absorção quase que completa do saco da gema, que podem ter estimulado o aumento na ingestão da dieta.

Quando se considerou o dia de nascimento da ave como o primeiro dia de idade, o consumo de ração foi maior após 24 horas de alojamento do que após 0 hora. O peso médio das aves não sofreu influência dos intervalos entre o nascimento e alojamento, pesquisados após 21 dias de nascimento ou alojamento.

O peso do saco vitelino diminuiu, linearmente, com o aumento no intervalo entre o nascimento e o alojamento em pintos, no experimento 2 (Tabela 7). Não há um consenso sobre qual a influência que o alojamento imediato exerce na utilização dos nutrientes do saco vitelino e sua redução (Maiorka et al., 2000). Romanoff \& Romanoff (1963) verificaram que o alojamento imediato torna a absorção mais lenta, enquanto Bierer \& Eleazer (1965) observaram efeito oposto, e segundo Moafi \& Atikison (1990), Murakami et al. (1992) e Baião et al. (1998), o tempo de alojamento não interfere nessa característica. De acordo com Maiorka et al. (2000), a controvérsia de dados obtidos em literatura é devido ao fator idade da matriz e presença do alimento. No presente estudo, quanto maior o tempo de privação de alimento e de água, menor o tamanho do saco vitelino em pintos originados de matrizes com 27 semanas de idade.

O comprimento do intestino e peso da ave sofreram influência do intervalo entre o nascimento e o alojamento, e tiveram os menores valores observados em aves que permaneceram 48 horas alojadas (Tabela 7). A privação de alimento e de água que o animal sofreu, no período em que permaneceu na embalagem, pode estar relacionada a esses resultados.

Houve interação entre o intervalo de alojamento e o fornecimento de eletrólito, para peso e densidade do intestino delgado (Tabela 8). Na ausência de eletrólito, o intestino delgado apresentou redução no peso e densidade, à medida que aumentou o intervalo entre nascimento e alojamento. A presença de eletrólito evitou a perda de massa intestinal, que pode ter corrido no
Tabela 8. Desdobramento das interações significativas para peso e densidade do intestino delgado, de pintos oriundos de matrizes com 27 semanas de idade, suplementados com hidratante, na fase pós-alojamento, 24 horas após o alojamento ${ }^{(1)}$.

\begin{tabular}{lccc}
\hline Eletrólitos & \multicolumn{3}{c}{ Intervalo de alojamento (horas) } \\
\cline { 2 - 4 } & 0 & 24 & 48 \\
\hline \multirow{3}{*}{ Ausência } & $3,18 \mathrm{Aa}$ & Peso do intestino delgado $(\mathrm{g})$ \\
Presença & $1,66 \mathrm{Ba}$ & $2,01 \mathrm{Aab}$ & $1,40 \mathrm{Ab}$ \\
\hline \multicolumn{4}{c}{ Densidade do intestino delgado $\left(\mathrm{mg} \mathrm{cm}^{-1}\right)$} \\
Ausência & $77,24 \mathrm{Aa}$ & $47,69 \mathrm{Aab}$ & $32,95 \mathrm{Ab}$ \\
Presença & $39,25 \mathrm{Ba}$ & $56,80 \mathrm{Aa}$ & $50,78 \mathrm{Aa}$ \\
\hline
\end{tabular}

(1)Médias seguidas de letras iguais, maiúsculas na coluna e minúsculas na linha, não diferem significativamente.

período de embalagem. Minerais fornecidos via solução eletrolítica promoveram a absorção de aminoácidos e glicose, o que pode estar relacionado à manutenção da integridade intestinal (Underwood \& Suttle, 2001).

\section{Conclusões}

1. Não se recomenda o uso de hidrante na fase préalojamento.

2. Eletrólitos podem ser utilizados em frangos de corte na fase pós-alojamento.

\section{Agradecimentos}

Ao CNPq, pelas bolsas concedidas.

\section{Referências}

BAIÃO, N.C.; AGUILAR, C.A.L. Impacto do tempo de alojamento de pinto de corte sobre a produção de frango. In: CONFERÊNCIA APINCO DE CIÊNCIA E TECNOLOGIA AVÍCOLAS, 2001, Campinas. Anais. Campinas: FACTA, 2001. p.125-140.

BAIÃO, N.C.; BORGES, F.M.O. Efeitos de hidratantes para pintos de corte no dia do alojamento sobre o desempenho de frangos. Arquivos Brasileiros de Medicina Veterinária, v.47, p.831-837, 1995.

BAIÃO, N.C.; CANÇADO, S.V. Efeito do intervalo entre o nascimento e o alojamento de pintos sobre o desempenho de frangos. Arquivos Brasileiros de Medicina Veterinária, v.50, p.191-194, 1998.

BAIÃO, N.C.; CANÇADO, S.V.; LÚCIO, C.G. Efeito do período de jejum entre o nascimento e o alojamento de pintos sobre a absorção do saco vitelino. Arquivos Brasileiros de Medicina Veterinária, v.50, p.473-474, 1998.

BATAL, A.B.; PARSON, C.M. Effect of fasting versus feeding oasis after hatching on nutrient utilization in chicks. Poultry Science, v.81, p.853-859, 2002. 
BIERER, B.W.; ELEAZER, T.H. Effect of feed and water deprivation on yolk utilization in chicks. Poultry Science, v.44, p.1608-1609, 1965.

CANÇADO, S.V. Efeito do intervalo entre o nascimento e o alojamento de pintos de corte sobre o desempenho, digestibilidade da ração, desenvolvimento do trato gastrintestinal e atividade da lipase. 1999. 52p. Tese (Doutorado) - Universidade Federal de Minas Gerais, Belo Horizonte.

CUNHA, W.C.P. Avaliação do peso inicial do pinto de corte e níveis de metionina na ração pré-inicial na digestibilidade, desempenho, rendimento de carcaça e viabilidade econômica. 2003. 60p. Dissertação (Mestrado) - Universidade Federal de Goiás, Goiânia.

DAGHIR, N.J.; JONES, R. Breeder and hatchery management. In: DAGHIR, N.J. (Ed.). Poultry production. Wallingford: CAB International, 1997. p.255-292.

LAURENTIZ, A.C.; ARIKI, J.; ARAÚJO, L.F.; BORGES, S.A.; ARAÚJO, C.S.S.; MAIORKA, A. Adaptações digestivas póseclosão. In: CONFERÊNCIA APINCO DE CIÊNCIA E TECNOLOGIA AVÍCOLAS, 2001, Campinas. Anais. Campinas: FACTA, 2001. p.141-151.

MAIORKA, A. Efeito dos níveis de proteína bruta e balanço eletrolítico sobre o desempenho de frangos de 1 a 7 dias de idade. Revista Brasileira de Ciência Avícola, v.2 (supl), p.4, 2000.

MAIORKA, A.; SANTIN, E.; FISHER, S.A.V.; BRUNO, L.D.G.; BOLELI, I.C.; MACARI, M. Desenvolvimento do trato gastrintestinal de embriões oriundos de matrizes pesadas de 30 a 60 semanas de idade. Revista Brasileira de Ciência Avícola, v.2, p.141147, 2000.

MOAFI, M.; ATIKISON, J.L. The effect of feed availability on spare yolk utilization by broiler chicks. Poultry Science, v.69, p.95, 1990.
MONGIN, P. Recent advances in dietary cation-anion balance: applications in poultry. Proceedings of the Nutrition Society, v.40, p.285-294, 1981.

MORAN JUNIOR, E.T.; REINHART, B.S. Yield quality and subsequent performance of poults removed from the hatcher using early-late and complete collection procedures. Poultry Science, v.59, p.1918-1924, 1980.

MURAKAMI, H.; AKIBA, Y.; HORIGUCHI, M. Growth utilization of nutrients in newly hatched chicks with or without removal of residual yolk. Growth Development and Aging, v.56, p.75-84, 1992.

PINCHASOV, Y.; NOY, Y. Comparison of post-hatch holding time and subsequent early performance of broiler chicks and turkey poults. British Poultry Science, v.34, p.11-12, 1993.

ROMANOFF, A.L.; ROMANOFF, A.J. The avian egg. $2^{\text {nd }}$ ed. New York: Jonh Wiley \& Sons, 1963. 918p.

SALAZAR, A. Are you willing to pay for the cost of high-quality chicks? Or do you prefer the more expensive alternative? Poultry International, v.2, p.17-19, 2003.

SAS INSTITUTE (Cary, Estados Unidos). SAS user's guide: statistics, version 8. Cary, 1998. 956p.

TANAKA, A.; XIN, H. Energetic, mortality, and body mass change of breeder chicks subjected to different post-hatch feed dosage. Transactions of the American Society of Agricultural Engineers, v.40, p.1457-1461, 1997.

UNDERWOOD, E.J.; SUTTLE, N.F. Mineral nutrition of livestock. 3.ed. New York: CABI Publishing, 2001. 624p.

UNI, Z. Methods for early nutrition and their potential. In: EUROPEAN SYMPOSIUM OF POULTRY NUTRITION, 2003, Lillehammer. Proceedings. Lillehamme: PSA, 2003. p.254-260.

XIN H.; LEE, K. Use of Aqua-Jel and feed for nutrient supply during long journey air transport of baby chicks. Transactions of the American Society of Agricultural Engineers, v.39, p.11231126, 1996.

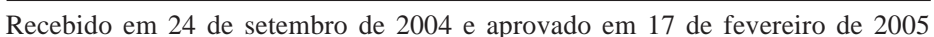

